\title{
Accelerating parabolic beams
}

\author{
Miguel A. Bandres \\ California Institute of Technology, Pasadena, California 91125, USA (bandres@caltech.edu)
}

Received May 23, 2008; accepted June 6, 2008;

posted June 20, 2008 (Doc. ID 96139); published July 22, 2008

\begin{abstract}
We demonstrate the existence of accelerating parabolic beams that constitute, together with the Airy beams, the only orthogonal and complete families of solutions of the two-dimensional paraxial wave equation that exhibit the unusual ability to remain diffraction-free and freely accelerate during propagation. Since the accelerating parabolic beams, like the Airy beams, carry infinite energy, we present exact finite-energy accelerating parabolic beams that still retain their unusual features over several diffraction lengths. (C) 2008 Optical Society of America
\end{abstract}

OCIS codes: $050.1940,260.1960,350.5500$.

Recently Siviloglou et al. [1-3] called the attention of the optics community by demostrating the existence of the accelerating Airy beams. These beams are finite-energy solutions of the paraxial wave equation that have the unusual ability to remain diffraction free while having a quadratic transverse shift during propagation over long distances. The pure Airy beams are perfectly diffractionless and have a quadratic transverse shift during propagation but cannot be physically realizable, because they have infinite energy. However, the Airy beams have finite energy and retain these unusual properties over long distances.

In this Letter, we introduce the accelerating parabolic beams and prove that they are, together with the two-dimensional Airy beams, the only orthogonal and complete family of explicit solutions of the twodimensional paraxial wave equation that remain diffraction free and freely accelerate during propagation. We also introduce a finite-energy version of the accelerating parabolic beams that can be physically realizable and exhibit the unusual properties of the ideal accelerating parabolic beams over a finite propagation distance.

We begin our analysis by considering the normalized paraxial wave equation $\nabla^{2} \psi+i \partial_{s} \psi=0$, where $\nabla^{2}$ $=\partial_{u u}+\partial_{v v},(u, v)=(x, y) / \kappa$ represents the dimensionless transverse coordinates, $\kappa$ is an arbitrary transverse scale, $s=z / 2 k \kappa^{2}$ is the normalized propagation distance, and $k$ is the wave number.

We know that the desired properties of the beam, that is, to remain diffraction free and to exhibit a transverse translation during propagation, can be expressed by

$$
|\psi(u, v, s)|=|\psi(u+\Delta(s), v, 0)|,
$$

where $\Delta(s)$ is a now-unknown function of $s$ that describes the transverse translation of the beam during propagation. The propagation of the beam $\psi(u, v, s)$ is formally given by

$$
\psi(u, v, s)=e^{-i s \mathbf{p}^{2}} \psi(u, v, 0),
$$

where $\mathbf{p}^{2}=-\nabla^{2}$.

For the one-dimensional case, in [4], Unnikrishnan et al. found that, if and only if $\Delta(s)=-s^{2}$, there is a unique decomposition of the evolution operator that achieves the desired conditions. Generalizing this work to the two-dimensional case, we find that the unique decomposition of the evolution operator is

$$
e^{-i s \mathbf{p}^{2}}=e^{-i 2 s^{3} / 3} e^{i s u} e^{-i s^{2} p_{u}} e^{-i s \hat{\mathrm{H}}},
$$

where

$$
\hat{\mathrm{H}}=-\left(\partial_{u}^{2}+\partial_{v}^{2}\right)+u
$$

and we have ignored any possible trivial rescaling and translation. From this unique decomposition we can see that the diffraction free and accelerating conditions given by Eq. (1) are satisfied if and only if $F(u, v)=\psi(u, v, 0)$ is a solution of

$$
\hat{\mathrm{H}} F(u, v)=\lambda F(u, v),
$$

and $\Delta(s)=-s^{2}$. Therefore, there exists a one-to-one mapping between accelerating solutions of the paraxial wave equation and solutions of the twodimensional lineal potential Schrödinger equation Eq. (5). This is important, because if we find all the solutions to the lineal potential Schrödinger equation we can find all the possible accelerating beams.

The two-dimensional lineal potential Schrödinger equation Eq. (5) is separable in an orthogonal coordinate system $(\xi, \eta)$ with metric $\mathbf{g}$ if $u(\xi, \eta) \sqrt{|\mathbf{g}|}$ can be expressed as $f(\xi)+h(\eta)$ for some functions $f$ and $h$; only the Cartesian and parabolic coordinate systems are known to have this property [5]. The separation in Cartesian coordinates corresponds to the twodimensional accelerating Airy beams [6] or a tensor product between an Airy beam in one coordinate and a plane wave in the other. To find solutions to $\hat{\mathrm{H}} F$ $=\lambda F$, notice that its solutions with eigenvalue $\lambda$ are given by $F_{0}(u-\lambda, v)$ where $F_{0}(u, v)$ is the $\lambda=0$ solution. Because $\hat{H}$ is a Hermitian operator, its eigenvalues $\lambda$ are real, i.e., $\lambda \in \mathbb{R}$ and its eigensolutions are orthogonal. Using the parabolic coordinates, which are defined by the transformation $(u, v)=\left(\eta^{2} / 2-\xi^{2} / 2, \eta \xi\right)$ where $\eta \in(-\infty, \infty), \quad \xi \in[0, \infty), F_{0}(\eta, \xi)=N(\eta) X(\xi)$; Eq. (5) with $\lambda=0$ separates into 


$$
\begin{aligned}
\left(-\partial_{\eta \eta} / 2+\eta^{4} / 4\right) N(\eta) & =E N(\eta), \\
\left(\partial_{\xi \xi} / 2+\xi^{4} / 4\right) X(\xi) & =E X(\xi) .
\end{aligned}
$$

Equation (6a) corresponds to the quartic oscillator equation [7], i.e., the one-dimensional Schrödinger equation with potential $V(\eta)=\eta^{4} / 4$ and $m=\hbar=1$. Square integrable eigensolutions of Eq. (6a), which we will denote by $\Theta_{n}(\eta)$, have discrete eigenvalues $E_{n}>0, n=0,1,2, \ldots$, with multiplicity one, are orthogonal, and have $n$ zeros. Because the quartic potential is parity invariant, the eigensolutions will have definite parity given by the parity of $n$. Notice that one can derive Eq. (6b) from Eq. (6a) by writing $i \xi$ for $\eta$, and vice versa. Therefore solutions to Eq. (6b) can be obtained by analytic continuation of the solutions of the quartic oscillator.

Finally, by collecting the partial results and rearranging terms, the accelerating parabolic beams are given by

$$
\psi_{n}(u, v, s, \lambda)=e^{i s\left(\eta^{2}-\xi^{2}\right) / 2+i s^{3} / 3} \Theta_{n}(\eta) \Theta_{n}(i \xi),
$$

where $\left(u-\lambda-s^{2}, v\right)=\left(\eta^{2} / 2-\xi^{2} / 2, \eta \xi\right), \quad n=0,1,2, \ldots$, and $\lambda \in \mathbb{R}$. Equation (7) is an exact solution of the paraxial wave equation that exhibits the unusual feature of remaining diffraction free and freely accelerates during propagation, and it represents the first important result of this Letter.

Several transverse field distributions of the loworder accelerating parabolic beams with $\lambda=0$ at $s=0$ are shown in Fig. 1. Accelerating parabolic beams with $\lambda \neq 0$ correspond to translations in the $u$ axis of the $\lambda=0$ beams. The beams have a definite parity respect to the $v$ axis, which is given by the parity of $n$. It is easy to observe the intrinsic parabolic geometry of the beams, i.e., all nodal lines are horizontal parabolas that open to the left or right. The order $n$ of the beams corresponds to the number of left horizontal parabolic nodal lines, and the width of the beam in the $v$ axis increases as $n$ increases.

The diffraction-free and freely accelerating properties of the parabolic beams are shown in Figs. 2(a) and 2(c)-2(e) for the zero-order beam; the high-order beams exhibit the same behavior. Notice that upon
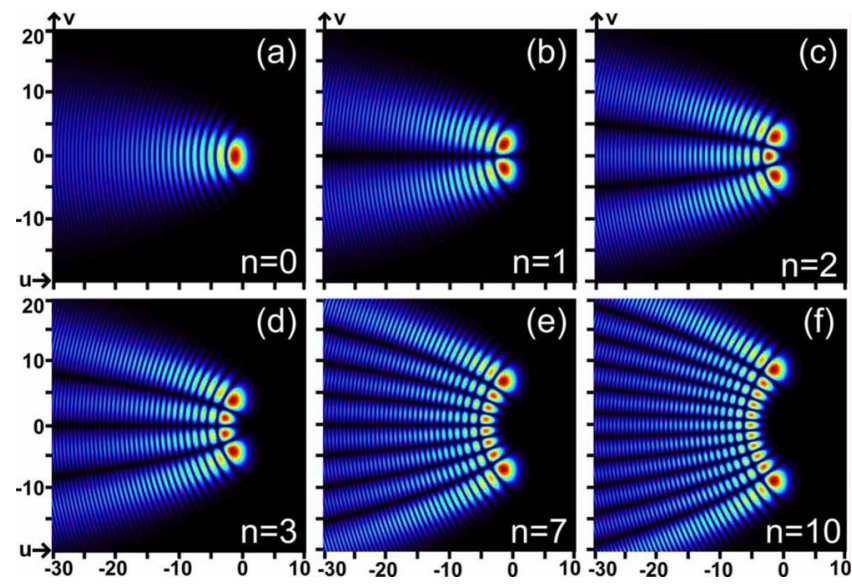

Fig. 1. (Color online) Transverse amplitude distributions of some accelerating parabolic beams with $\lambda=0$ at $s=0$. propagation the transverse field distribution translates in the $u$ axis without diffracting [Figs. 2(c)-2(e)] and that this translation is given by $s^{2}$, i.e., the beam has a ballistic lateral shift [Fig. 2(a)].

Because the map between solutions of Eq. (5) and the accelerating solutions is given by the unitary evolution operator, the orthogonality properties of the former solutions will be mapped to the latter. Therefore, the accelerating parabolic beams satisfy the following orthogonality condition:

$$
\iint_{-\infty}^{+\infty} \psi_{n}(u, v, s, \lambda) \psi_{m}(u, v, s, \gamma) \mathrm{d} u \mathrm{~d} v \propto \delta_{n m} \delta(\lambda-\gamma) .
$$

The accelerating parabolic beams have infinite extent and carry infinite energy owing to the asymptotic behavior of $\Theta_{n}(i \xi) \sim \sin \left(\sqrt{2} \xi^{3} / 6+\pi / 6\right) / \xi$ as $\xi \rightarrow \infty$; therefore, they are not physically realizable. Their infinite oscillating tail that extends in the negative $u$ axis can be appreciated in Figs. 1 and 2(c)-2(e).

The construction of finite-energy solutions is straightforward if we use the symmetries of the paraxial wave equation. The paraxial wave equation is invariant under time and space translations; physically, this means that it is invariant on the position of the origin of our coordinate system. However, mathematically these translations are not restricted to be real and can be complex. Therefore, if we change $s \rightarrow s-i a$ and $u \rightarrow u-a^{2}$ in Eq. (7) we get a solution of the paraxial wave equation with a profile at the input plane $(s=0)$ given by

$$
\phi_{n}(u, v, 0, \lambda) \propto e^{a u} \Theta_{n}(\eta) \Theta_{n}(i \xi)
$$

where $(u-\lambda, v)=\left(\eta^{2} / 2-\xi^{2} / 2, \eta \xi\right)$. If we chose $\operatorname{Re}(a)$ $>0$ the exponential function will ensure the containment of the infinite tail of the accelerating parabolic beam. Then the finite-energy accelerating parabolic beams are given by

$$
\phi_{n}(u, v, s, \lambda)=e^{i(s-i a)\left(\eta^{2}-\xi^{2}\right) / 2} e^{i(s-i a)^{3} / 3} \Theta_{n}(\eta) \Theta_{n}(i \xi),
$$

where

$$
\left(u-\left(\lambda+a^{2}\right)-(s-i a)^{2}, v\right)=\left(\eta^{2} / 2-\xi^{2} / 2, \eta \xi\right) .
$$

Equation (9) is an exact solution of the paraxial wave equation with finite energy that retains the diffraction-free and transverse acceleration features over several diffraction lengths, and it represents the second important result of this Letter.

It is important to note that because the factor $\left(\lambda+a^{2}\right)+(s-i a)^{2}$ in the left-hand side of Eq. (10) is complex, the parabolic coordinates $\eta(s)$ and $\xi(s)$ now take values in the complex plane. This means that the initial field distribution at $s=0$ changes shape (i.e., diffracts) as it propagates, because the functions $\Theta_{n}$ are now evaluated in different complex values at each transverse plane. To have a quasi-diffractive behavior we want to avoid this as much as possible; therefore, the finite accelerating parabolic beams 

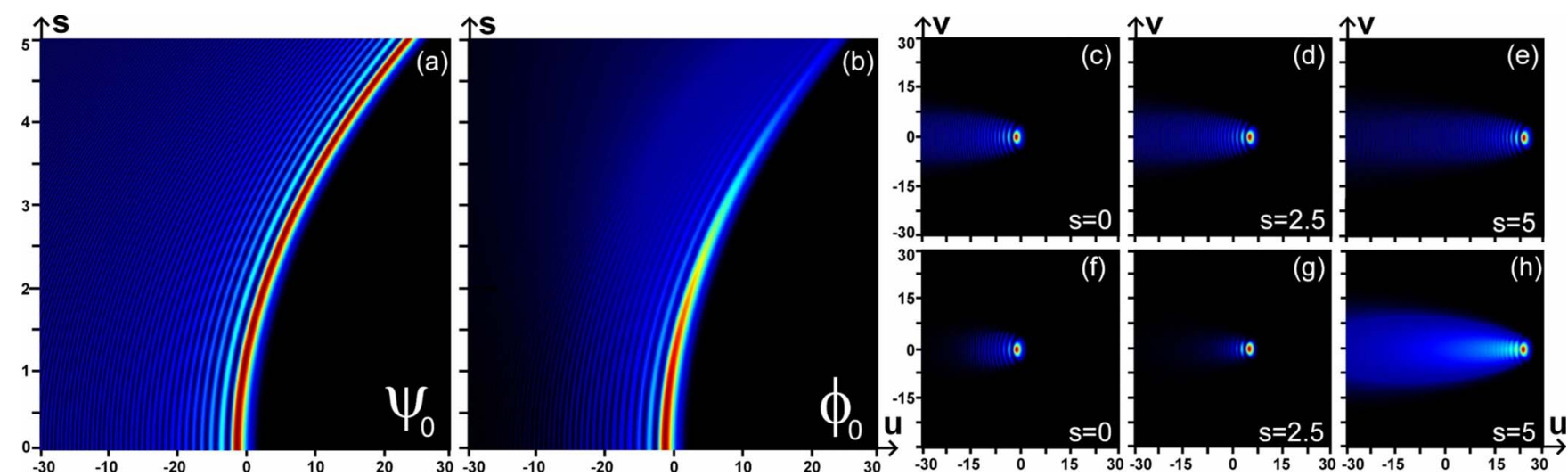

Fig. 2. (Color online) (a), (b) Propagation dynamics and (c)-(e), (f)-(h) transverse intensity distributions at different $s$ planes of the zero-order diffraction-free accelerating parabolic beam and zero-order finite-energy accelerating parabolic beam $(a=0.05)$, respectively.

have a quasi-diffractive behavior if $a \ll 1$. Notice that when $a=0$ we recover the ideal diffraction-free accelerating parabolic beams.

Figures 2(b) and 2(f)-2(h) show the propagation dynamics of the zero-order finite accelerating parabolic beam with $a=0.05$. The high-order finite beams with the same parameters exhibit a similar behaivor. As clearly seen, for $a \ll 1$ the beam still displays all the interesting characteristics of the ideal accelerating parabolic beam. During propagation, it remains quasi-invariant for $s \leqslant 3$. More importantly, in spite of the exponential truncation, the finite accelerating parabolic beams still exhibit a quadratic lateral shift. However the expected value of $u$ (i.e., the centroid of the beam), $\langle u(s)\rangle=\iint u\left|\phi_{n}\right|^{2} \mathrm{~d} u \mathrm{~d} v / \iint\left|\phi_{n}\right|^{2} \mathrm{~d} u \mathrm{~d} v$, of a finite-energy accelerating parabolic beam can be defined, and it is given by

$$
\langle u(s)\rangle=\langle u(0)\rangle+2 \operatorname{Im}(a) s,
$$

where $\langle u(0)\rangle$ is the centroid at $s=0$, and $\langle v(s)\rangle=0$ by parity. Therefore, as for the finite-energy Airy beam $[3,8,9]$, the propagation of the accelerating parabolic
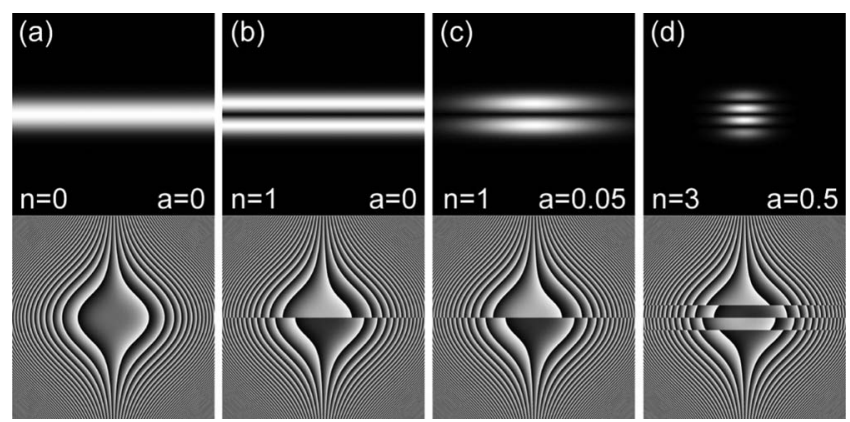

Fig. 3. Amplitude (upper row) and phase (bottom row) of the Fourier spectrum of several accelerating parabolic beams with $\lambda=0$. beam's centroid follows exactly the expected geometrical path. Also, the accelerating parabolic beams follow the same transformation laws as the Airy beams while propagating through $\mathrm{ABCD}$ optical systems [9].

The Fourier spectrum of the finite-energy accelerating parabolic beams at the plane $z=0$ [Eq. (8)] is given by

$$
\mathcal{F}\left[\phi_{n}(\lambda)\right]\left(k_{u}, k_{v}\right) \propto i^{n} e^{i\left(k_{u}+i a\right)\left(k_{v}^{2}-\lambda\right)} e^{i\left(k_{u}+i a\right)^{3} / 3} \Theta_{n}\left(\sqrt{2} k_{v}\right),
$$

and it is depicted in Fig. 3. In [10] accelerating parabolic beams were generated experimentaly by encoding the amplitude and phase of its Fourier spectrum onto a liquid-crystal display.

We acknowledge support from the Secretaría de Educación Pública de México.

\section{References}

1. G. A. Siviloglou and D. N. Christodoulides, Opt. Lett. 32, 979 (2007).

2. G. A. Siviloglou, J. Broky, A. Dogariu, and D. N. Christodoulides, Phys. Rev. Lett. 99, 213901 (2007).

3. G. A. Siviloglou, J. Broky, A. Dogariu, and D. N. Christodoulides, Opt. Lett. 33, 207 (2008).

4. K. Unnikrishnan and A. R. P. Rau, Am. J. Phys. 64, 1034 (1996).

5. C. P. Boyer, E. G. Kalnins, and J. W. Miller, J. Math. Phys. 16, 499 (1975).

6. I. M. Besieris, A. M. Shaarawi, and R. W. Ziolkowski, Am. J. Phys. 62, 519-521 (1994).

7. K. Banerjee, S. P. Bhatnagar, V. Choudhry, and S. S. Kanwal, Proc. R. Soc. London 360, 575 (1978).

8. I. M. Besieris and A. M. Shaarawi, Opt. Lett. 32, 2447 (2007).

9. M. A. Bandres and J. C. Gutiérrez-Vega, Opt. Express 15, 16719 (2007).

10. J. A. Davis, M. J. Mitry, M. A. Bandres, and D. M. Cottrell, "Observation of accelerating parabolic beams," Opt. Express (to be published). 